\title{
Correction: Efficacy of selective laser trabeculoplasty in primary angle closure disease
}

Srishti Raj • Basavraj Tigari • T. T. Faisal • Natasha Gautam • Sushmita Kaushik • Parul Ichhpujani • Surinder S. Pandav · Jagat Ram

Published online: 10 July 2020

(c) The Author(s), under exclusive licence to The Royal College of Ophthalmologists 2020

Correction to: Eye

https://doi.org/10.1038/s41433-018-0165-5

The original Article contained a key error in the methodology section: in the sentence beginning with
"For patients on antiglaucoma treatment", the authors accidentally mentioned "laser iridotomy" instead of "SLT". This has been corrected in the PDF and HTML versions of this article. 